\title{
On feedforward control of piezoelectric dual-stage actuator systems
}

\author{
Clarisse Pétua Bosman Barros, Hans Butler, Jeroen van de Wijdeven, Roland Tóth
}

\begin{abstract}
The feedforward control design problem for a single-axis dual-stage actuator system with piezoelectric actuator at the short-stroke is analyzed in this paper. With such actuator layout, the main question is how to balance the contribution of the individual actuators in a efficient manner, while complying to actuators limitations. A control configuration and a sequential design methodology are proposed to take into account interactions between actuators. In addition, various feedforward controller design strategies that conform to the configuration proposed are presented, such as inversion based feedforward, mass feedforward and standard compliance compensation. Based on observed shortcomings of each feedforward design, a novel mixed compliance compensation feedforward controller is presented. Results are analyzed in terms of their physical interpretations and simulation studies.
\end{abstract}

\section{INTRODUCTION}

Lithography machines are the current standard for producing microchips in a cost-effective manner in the semiconductor industry. A golden rule, known as Moore's law, states that the number of transistors in a dense integrated circuit is expected to double every two years [1] and this prediction has set the pace for innovation and development in lithography for over 50 years [2], [3]. The wafer stage ${ }^{1}$ is the motion system which is responsible for positioning the silicon wafer under a lens during exposure, currently providing nanometer accuracy. Development of this part of the machine is essential to continue at the current pace.

High-accuracy, high-speed and wide-range motion systems like wafer stages are conventionally equipped with a dualstage actuator (DSA) system to provide a large-distance range with nanometer positioning accuracy [5]. The longstroke actuator is responsible for moving the stage over the necessary range, but with the current technology it is not possible to achieve the target accuracy and a short-stroke stage is added to this end. Piezoelectric actuators (in short, piezoactuators) are strong candidates for short-stroke positioning of the wafer table in the next generation of lithography systems due to their sub-nanometer positioning accuracy, rapid response speed, large blocking force and compact structure [6]. They are widely used in high-performance microscopes, but their use as a wafer positioning system is challenging and leads to imperative specialized designs for the motion control system.

C.P. Bosman Barros, H. Butler and R. Tóth are with the Eindhoven University of Technology, Department of Electrical Engineering, Control Systems Group, Eindhoven, The Netherlands. Email of the corresponding author: c.p.bosman.barrosetue.nl. H. Butler and J. van de Wijdeven are with ASML, 5504 DR Veldhoven, The Netherlands. R. Tóth is also with the Systems and Control Laboratory, Institute for Computer Science and Control, Kende u. 13-17, H-1111 Budapest, Hungary.

${ }^{1}$ Refer to [4], [3] for more information on lithography machines, wafer scanners and how this systems operate.
In current wafer stages, as mentioned in [7], feedforward control forces are responsible for more than $99 \%$ of the associated performance. Specialized reference feedforward control is then essential for fulfilling high accuracy with high production throughput requirements [8]. In particular for short-stroke positioning with piezo-actuator, a stiff connection between the stage elements is present and leads to interactions between actuator actions that can seriously deteriorate the control system performance when not considered in the design stage. In addition, the high production throughput results in high accelerations and aggressive motion profiles, which brings strict demands on actuators, i.e., it pushes them to their limits. Complying with these limits has to be ensured by the feedforward control system.

The feedforward control problem for DSA positioners primarily incorporates the task of balancing the contribution of the individual actuators. As these systems have two actuators for a single-axis motion, any given trajectory may be achieved by different feedforward controllers with complementary combinations of actuators actions. The major challenge is then to synthesize a controller that best utilizes each actuator while satisfying their physical limitations.

The most direct approach is to design a feedforward controller that equals the inverse of the plant, theoretically leading to perfect reference tracking [9], [10], [8]. Such a controller can be calculated based on the pseudo-inverse of the transfer matrix, but for DSA systems with piezo-actuators at short-stroke it results in a controller without physical interpretation and, generally, with inefficient separation for each actuator action. Another common strategy is to apply a decoupling filter for the system and calculate the feedforward controller based on the decoupled system [11]. However, for the cases at which the interactions between the components of the system are severe, the obtained forces do not reflect the actual forces at the input of the plant. Thus, it becomes complex to foresee if each actuator is being used efficiently and how to re-design to conform to limits.

In this paper, we present a feedfoward control configuration and a systematic procedure to design feedforward controllers for single-axis motion DSA systems (Contribution C1). Then, an investigation and comparison of various feedforward design techniques in the considered configuration is performed (Contribution C2). Specifically for the wafer stage application, a novel design named mixed-compliance compensation is proposed to overcome observed shortcomings of each presented feedforward design (Contribution C3). Its superior performance is demonstrated in terms of a simulation study.

The paper is organized as follows. The considered DSA 


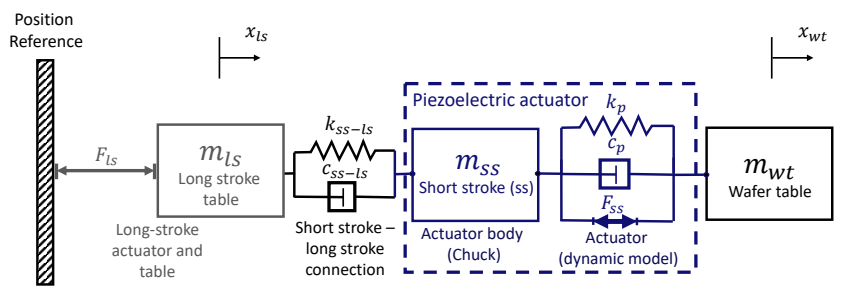

Fig. 1. Schematic view of the plant. Three mass system with two input forces, $F_{\mathrm{ss}}$ and $F_{\mathrm{ls}}$, long-stroke table position $x_{\mathrm{ls}}$ and wafer table position (performance target) $x_{\mathrm{wt}}$.

TABLE I

VALUES OF THE COEFFICIENTS USED IN THE MODEL

\begin{tabular}{|c|c|c|c|c|c|c|}
\hline$m_{\mathrm{wt}}$ & $m_{\mathrm{ss}}$ & $m_{\mathrm{ls}}$ & $k_{\mathrm{p}}$ & $c_{\mathrm{p}}$ & $k_{\mathrm{ss}-\mathrm{ls}}$ & $c_{\mathrm{ss}-\mathrm{ls}}$ \\
\hline $1 \mathrm{~kg}$ & $5 \mathrm{~kg}$ & $80 \mathrm{~kg}$ & $10^{8} \frac{\mathrm{N}}{\mathrm{m}}$ & $200 N \cdot \frac{\mathrm{s}}{\mathrm{m}}$ & $10^{8} \frac{\mathrm{N}}{\mathrm{m}}$ & $490 \mathrm{~N} \cdot \frac{\mathrm{s}}{\mathrm{m}}$ \\
\hline
\end{tabular}

system model and problem statement are presented in Section II. In Section III, the sequential feedforward control design procedure is presented (C1). Then, in Section IV, the various design options for feedforward control are presented, compared, and validated in continuous-time simulations (C2 and C3). Finally, in Section V, concluding remarks are given.

Notation: $\mathbb{C}$ denotes the set of complex numbers and $\mathbb{R}$ the set of real numbers. $C^{n}(0, \infty)$ denotes the class of functions on $(0, \infty)$ that are $n$-times continuously differentiable. The time is denoted by $t \in \mathbb{R}$ and $s \in \mathbb{C}$ is the complex frequency (Laplace variable).

\section{PROBLEM STATEMENT}

The single-axis motion model considered is presented in this section, followed by a formal definition for the feedforward control design problem and the proposed control system configuration.

Wafer stage model. The schematic of the DSA system is depicted in Figure 1. The wafer table is considered as a mass $m_{\mathrm{wt}}$. The piezo-actuator is modeled as a mass $m_{\mathrm{ss}}$ in series with its actuation model $\left(k_{\mathrm{p}}\right.$ is the stiffness, $c_{\mathrm{p}}$ is the damping coefficient and $F_{\mathrm{ss}}$ is the force created by the inverse piezoelectric effect). The connection between the long-stroke and the short-stroke movers is made of other material with relatively low mass and modeled as an spring with stiffness $k_{\mathrm{ss}-\mathrm{ls}}$ in parallel to a damper with coefficient $c_{\mathrm{ss}-\mathrm{ls}}$ (KelvinVoigt model for solids). Lastly, the long-stroke actuator and table are modeled as a force $F_{\mathrm{ls}}$ and a mass $m_{\mathrm{ls}}$.

The modeling of piezoelectric actuator dynamics is based on physical principles, according to knowledge of the material characteristics and the piezoelectric constitutive equations [12], explained in details in [6], [13]. For this paper, we assume that the nonlinear behavior is compensated at the electrical domain by, for instance, a charge amplifier. The long-stroke actuator can be a planar motor or a $\mathrm{H}$ configuration motor [14], and its dynamics is modeled simply as a force source. As a result, the model based on Figure 1 is linear.

The target performance position is the wafer table position $y(t)=x_{\mathrm{wt}}(t)$ and the input of the system is $u(t)=\left[\begin{array}{ll}F_{\mathrm{ss}}(t) & F_{\mathrm{ls}}(t)\end{array}\right]^{\top}$. Solving the equations of motion and taking the Laplace transform, the transfer functions are

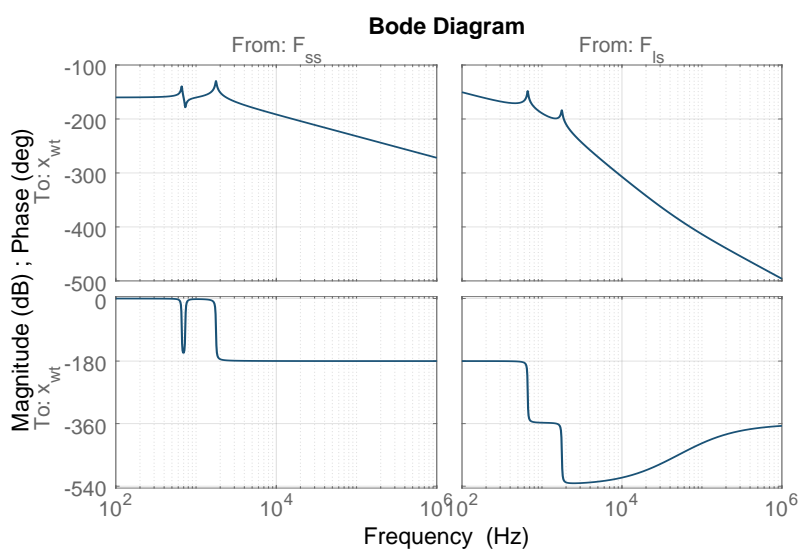

Fig. 2. Bode plot of $P_{\mathrm{wt}-\mathrm{ss}}(s)$ (left) and $P_{\mathrm{wt}-\mathrm{ls}}(s)$ (right).

TABLE II

ABSOLUTE VALUES FOR LIMITS OF ACTUATORS

\begin{tabular}{|c|c|c|c|c|}
\hline$v_{\max }$ & $a_{\max }$ & $j_{\max }$ & $s_{\max }$ & $F_{\mathrm{ss}-\max }$ \\
\hline $1 \mathrm{~m} / \mathrm{s}$ & $100 \mathrm{~m} / \mathrm{s}^{2}$ & $2 * 10^{4} \mathrm{~m} / \mathrm{s}^{2}$ & $2 * 10^{7} \mathrm{~m} / \mathrm{s}^{2}$ & $1500 \mathrm{~N}$ \\
\hline
\end{tabular}

$$
x_{\mathrm{wt}}(s)=\left[\begin{array}{ll}
P_{\mathrm{wt}-\mathrm{ss}}(s) & P_{\mathrm{wt}-\mathrm{ls}}(s)
\end{array}\right]\left[\begin{array}{c}
F_{\mathrm{ss}}(s) \\
F_{\mathrm{ls}}(s)
\end{array}\right],
$$

with

$$
\begin{gathered}
P_{\mathrm{wt}-\mathrm{ss}}(s)=\left(m_{\mathrm{ss}}+m_{\mathrm{ls}}\right) \frac{\frac{m_{\mathrm{ss}} m_{\mathrm{ls}}}{m_{\mathrm{ss}}+m_{\mathrm{ls}}} s^{2}+c_{\mathrm{ss}-\mathrm{ls}} s+k_{\mathrm{ss}-\mathrm{ls}}}{d(s)} \\
P_{\mathrm{wt}-\mathrm{ls}}(s)=\frac{\left(c_{\mathrm{p}} s+k_{\mathrm{p}}\right)\left(c_{\mathrm{ss}-1 \mathrm{~s}} s+k_{\mathrm{ss}-1 \mathrm{~s}}\right)}{s^{2} d(s)} \\
d(s)=m_{\mathrm{wt}} m_{\mathrm{ss}} m_{\mathrm{ls}} s^{4}+\left(c_{\mathrm{ss}-\mathrm{ls}} m_{\mathrm{wt}}\left(m_{\mathrm{ss}}+m_{\mathrm{ls}}\right)\right. \\
\left.+c_{\mathrm{p}} m_{\mathrm{ls}}\left(m_{\mathrm{wt}}+m_{\mathrm{ss}}\right)\right) s^{3}+\left(k_{\mathrm{ss}-\mathrm{ls}} m_{\mathrm{wt}}\left(m_{\mathrm{ss}}+m_{\mathrm{ls}}\right)\right. \\
\left.+k_{\mathrm{p}} m_{\mathrm{ls}}\left(m_{\mathrm{wt}}+m_{\mathrm{ss}}\right)+c_{\mathrm{p}} c_{\mathrm{ss}-\mathrm{ls}}\left(m_{\mathrm{wt}}+m_{\mathrm{ss}}+m_{\mathrm{ls}}\right)\right) s^{2} \\
+\left(m_{\mathrm{wt}}+m_{\mathrm{ss}}+m_{\mathrm{ls}}\right)\left(c_{\mathrm{p}} k_{\mathrm{ss}-1 \mathrm{~s}}+c_{\mathrm{ss}-\mathrm{ls}} k_{\mathrm{p}}\right) s \\
+\left(m_{\mathrm{wt}}+m_{\mathrm{ss}}+m_{\mathrm{ls}}\right) k_{\mathrm{p}} k_{\mathrm{ss}-\mathrm{ls}} .
\end{gathered}
$$

The Laplace transform of any signal $*(t)$ is noted by $*(s)$, considering its appropriate region of convergence. Table I contains the values for the coefficients which are used onward and Figure 2 depicts the Bode plot from $F_{\mathrm{ss}}$ and $F_{\text {ls }}$ to $x_{\mathrm{wt}}$.

Limitations. The considered limits are present in Table II. The long-stroke actuator has no range constraints, but has velocity $\left(v_{\max }[\mathrm{m} / \mathrm{s}]\right)$, acceleration $\left(a_{\max }\left[\mathrm{m} / \mathrm{s}^{2}\right]\right)$, jerk $\left(j_{\max }\left[\mathrm{m} / \mathrm{s}^{3}\right]\right)$ and $\operatorname{snap}\left(s_{\max }\left[\mathrm{m} / \mathrm{s}^{4}\right]\right)$ limits. The force $F_{\mathrm{ss}}$ is the force created by the inverse piezoelectric effect, and it is also bounded. Observe that this indicates the maximum elongation/compression the piezo-actuator can actively perform: for no external forces acting on it, it equals $F_{\mathrm{ss}-\max } / k_{\mathrm{p}}=15 \mu \mathrm{m}$.

Control system configuration. A schematic view of the overall control system is depicted in Figure 3. The plant model, which also contains the measurement of the longstroke table position $x_{\mathrm{ls}}$, is given by

$$
\left[\begin{array}{c}
x_{\mathrm{wt}}(s) \\
x_{\mathrm{ls}}(s)
\end{array}\right]=\left[\begin{array}{cc}
P_{\mathrm{wt}-\mathrm{ss}}(s) & P_{\mathrm{wt}-\mathrm{ls}}(s) \\
P_{\mathrm{ls}-\mathrm{ss}}(s) & P_{\mathrm{ls}-\mathrm{ls}}(s)
\end{array}\right]\left[\begin{array}{c}
F_{\mathrm{ss}}(s) \\
F_{\mathrm{ls}}(s)
\end{array}\right],
$$

where $P_{\mathrm{ls}-\mathrm{ss}}$ and $P_{\mathrm{ls}-\mathrm{ls}}$ are, respectively, the transfer functions from the forces inputs $F_{\mathrm{ss}}$ and $F_{\mathrm{ls}}$ to $x_{\mathrm{ls}}$. 


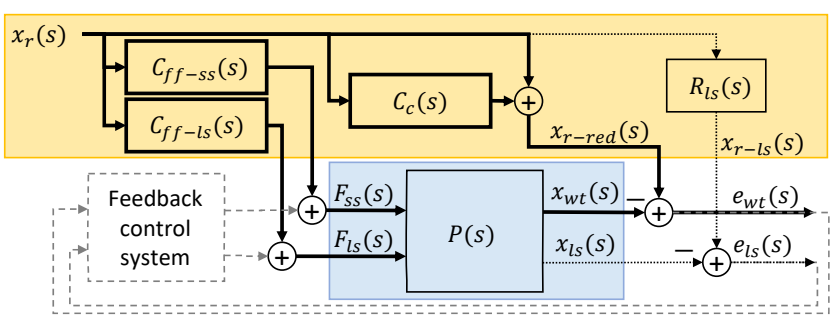

Fig. 3. Schematic view of the control system.

The feedforward control system is composed by the controller $C_{\mathrm{ff}}(s)=\left[\begin{array}{ll}C_{\mathrm{ff}-\mathrm{ss}}(s) & C_{\mathrm{ff}-\mathrm{ls}}(s)\end{array}\right]^{\top}$ and two reference alignment components: $R_{\mathrm{ls}}(s)$ and $C_{\mathrm{c}}(s) . C_{\mathrm{ff}-\mathrm{ss}}(s)$ and $C_{\mathrm{ff}-\mathrm{Is}}(s)$ are feedforward components regarding the shortstroke and long-stroke actuators, respectively. The reference and error signals for the long-stroke and wafer tables used by the feedback controller are defined respectively as $x_{\mathrm{r}-\mathrm{ls}}(s)=$ $R_{\mathrm{ls}}(s) x_{\mathrm{r}}(s), x_{\mathrm{r}-\mathrm{red}}(s)=\left(1+C_{\mathrm{c}}(s)\right) x_{\mathrm{r}}(s), e_{\mathrm{ls}}(s)=R_{\mathrm{ls}}(s) x_{\mathrm{r}}(s)-$ $x_{\mathrm{ls}}(s)$ and $e_{\mathrm{wt}}(s)=\left(1+C_{\mathrm{c}}(s)\right) x_{\mathrm{r}}(s)-x_{\mathrm{wt}}(s)$.

The actions arising out of the feedforward system needs to be aligned to the feedback control system and this alignment is performed by reference re-design through the filter $R_{\mathrm{ls}}(s)$ (detailed in Section III). For $x_{\mathrm{wt}}(t)$, there are moments at which the position of the wafer table can deviate in a specific manner from the initial reference signal, this allowed deviation being described by $C_{\mathrm{c}}(s)$ (detailed in Section IV).

Feedforward control design problem. A feedforward controller defined in the format of a transfer matrix $C_{\mathrm{ff}}(s)$, $C_{\mathrm{ff}}: \mathbb{C} \rightarrow \mathbb{P}_{C} \subseteq \mathbb{C}^{2 \times 1}$, such that $u(s)=C_{\mathrm{ff}}(s) x_{\mathrm{r}}(s)$, needs to be designed such that the performance output $y$ tracks the reference trajectory $x_{\mathrm{r}}$ (known in advance). In addition, the filter $R_{\mathrm{ls}}(s)$ needs to be designed to have the reference $x_{\mathrm{r}-\mathrm{ls}}$ for $x_{\mathrm{ls}}$ accordingly to the designed $C_{\mathrm{ff}}(s)$.

\section{SEQUENTIAL FEEDFORWARD DESIGN PROCEDURE}

This section presents a sequential design procedure for the feedforward controller $C_{\mathrm{ff}}(s)$, which together with the configuration in Figure 1 constitute Contribution C1. By the proposed procedure, first the long-stroke actuator feedforward component is designed, given that it is the actuator without range-restrictions, followed by the designed of the short-stroke actuator feedforward component. In this way, the interactions between actuators actions in the system are incorporated in the design phase in an intuitive manner.

Procedure: sequential feedforward controller design.

1) Define reference trajectory's order.

2) Design $C_{\mathrm{ff}-\mathrm{ls}}(s)$.

3) Calculate the remaining forces needed for perfect tracking.

4) Design $C_{\mathrm{ff}-\mathrm{ss}}(s)$, based on the expression obtained in the Step 3.

5) Based on the overall feedforward controller, perform the reference design for the long-stroke table (design $R_{\mathrm{ls}}(s)$ ) and, possibly, for the wafer table (design $C_{\mathrm{c}}(s)$ ).

The $C_{\mathrm{c}}(s)$ refers to a reference compliance compensation technique presented in section IV. If not applied, $C_{\mathrm{c}}(s)=0$ and $x_{\mathrm{r}-\text { red }}(t)=x_{\mathrm{r}}(t)$. The steps 1,3 and 5 are generic and explained further next.

Step 1. The reference order of the trajectory is defined here as the higher derivative of the position reference $x_{\mathrm{r}}$ which is known and bounded. Given that the the reference signal is of $n$-th order and by defining $d_{\mathrm{r}}(s)=s^{n} x_{\mathrm{r}}(s)$, we have that

$$
u(s)=C_{\mathrm{ff}}(s) x_{\mathrm{r}}(s)=\frac{C_{\mathrm{ff}}(s)}{s^{n}} d_{\mathrm{r}}(s) .
$$

Consequently, if at the implementation phase the $d_{\mathrm{r}}$ is used as the controller input and $\frac{C_{\mathrm{ff}}(s)}{s^{n}}$ as the controller, it is pertinent to design non-causal controllers $C_{\mathrm{ff}}(s)$ with numerator-todenominator relative order less or equal to $n$.

Step 3. Consider $C_{\mathrm{ff}-\mathrm{ls}}(s)$ is already synthesized. For the target application, the expression for the remaining forces needed for perfect tracking is obtained as follows. From (1), we can derive:

$$
x_{\mathrm{wt}}(s)=P_{\mathrm{wt}-\mathrm{ss}}(s) F_{\mathrm{ss}}(s)+P_{\mathrm{wt}-\mathrm{ls}}(s) C_{\mathrm{ff}-\mathrm{ls}}(s) x_{\mathrm{r}}(s) .
$$

In order to achieve perfect tracking, we need to have $x_{\mathrm{wt}}=x_{\mathrm{r}}$ and, consequently,

$$
x_{\mathrm{r}}(s)=P_{\mathrm{wt}-\mathrm{ss}}(s) F_{\mathrm{ss}}(s)+P_{\mathrm{wt}-\mathrm{ls}}(s) C_{\mathrm{ff}-\mathrm{ls}}(s) x_{\mathrm{r}}(s) .
$$

Thus, the necessary force to be provided by the piezoactuator needs to be equal to

$$
F_{\mathrm{ss}}(s)=\left(\frac{1}{P_{\mathrm{wt}-\mathrm{ss}}(s)}-\frac{P_{\mathrm{wt}-\mathrm{ls}}(s) C_{\mathrm{ff}-\mathrm{ls}}(s)}{P_{\mathrm{wt}-\mathrm{ss}}(s)}\right) x_{\mathrm{r}}(s),
$$

leading to

$$
C_{\mathrm{ff}-\mathrm{ss}}(s)=\left(\frac{1}{P_{\mathrm{wt}-\mathrm{ss}}(s)}-\frac{P_{\mathrm{wt}-\mathrm{ls}}(s) C_{\mathrm{ff}-\mathrm{ls}}(s)}{P_{\mathrm{wt}-\mathrm{ss}}(s)}\right) .
$$

Observe that by designing the short-stroke feedforward component based on (5), the long-stroke actuator contribution for the reference tracking is included in $-P_{\mathrm{wt}-\mathrm{ls}}(s) C_{\mathrm{ff}-\mathrm{ls}}(s)$ and the corresponding interaction of the actuators is given by its multiplication to $\left(P_{\mathrm{wt}-\mathrm{ss}}(s)\right)^{-1}$.

Step 5. Finally, it is important to prevent the feedback control system from counteracting the feedforward control actions, specially when aiming to achieve fast settling time (directly related to achievable throughput of the machine). In order to align the feedback and the feedforward control systems, the reference for the feedback controller $\left(x_{\mathrm{r}-\mathrm{ls}}\right)$ needs to be aligned with what the feedforward controller is commanding for the system i.e. $u(s)=C_{\mathrm{ff}}(s) x_{\mathrm{r}}(s)$. Thus, the reference of the long-stroke table $x_{\mathrm{r}-\mathrm{ls}}$ is given by

$$
x_{\mathrm{r}-\mathrm{ls}}(s)=\left[\begin{array}{ll}
P_{\mathrm{ls}-\mathrm{ss}}(s) & P_{\mathrm{ls}-\mathrm{ls}}(s)
\end{array}\right]\left[\begin{array}{l}
C_{\mathrm{ff}-\mathrm{ss}}(s) \\
C_{\mathrm{ff}-\mathrm{ls}}(s)
\end{array}\right] x_{\mathrm{r}}(s),
$$

resulting in

$$
R_{\mathrm{ls}}(s)=\left[P_{\mathrm{ls}-\mathrm{ss}}(s) \quad P_{\mathrm{ls}-\mathrm{ls}}(s)\right] C_{\mathrm{ff}}(s)
$$

and this is a fixed filter with no design freedom.

The presented procedure can be applied to different combinations of actuator types besides the one in focus and extended to feedforward design of systems with more actuators in the same motion-axis. The calculated feedforward forces are the ones that are going to be applied directly by the actuators and hence fulfillment of limits can be checked directly after steps 2 and 4 . If the check at any of these 

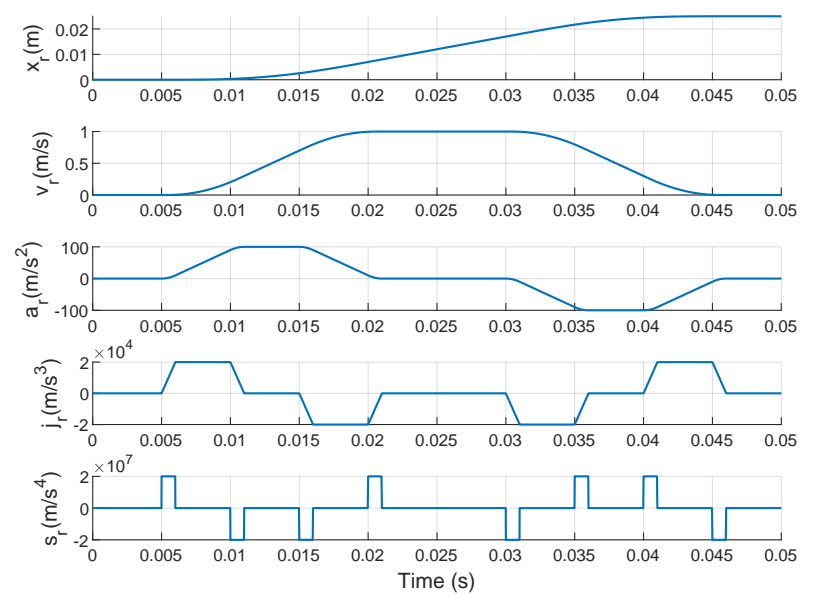

Fig. 4. Fourth-order position trajectory signals: position $\left(x_{\mathrm{r}}(t)\right)$, velocity $\left(v_{\mathrm{r}}(t)\right)$, acceleration $\left(a_{\mathrm{r}}(t)\right)$, jerk $\left(j_{\mathrm{r}}(t)\right)$ and snap $\left(s_{\mathrm{r}}(t)\right)$.

steps shows that a limitation is not respected, then only the component designed at that step needs to be re-designed.

Next, different feedforward control systems for the wafer stage are synthesized and compared, following the presented procedure and control configuration.

\section{FEEDFORWARD CONTROL SYSTEM DESIGNS}

The feedforward control design problem to the wafer stage is investigated in this section and constituted Contribution C2. Six feedforward strategies are presented and analyzed considering their applicability and their physical interpretation along with continuous-time simulations. For the initial four designs, there is no re-design of the wafer table reference (i.e., $C_{\mathrm{c}}(s)=0 \rightarrow x_{\mathrm{r}-\text { red }}(t)=x_{\mathrm{r}}(t)$ ). In the sequel, the reference signal used in time simulations is defined, then the feedforward controllers are presented and at the last step they are compared.

The feedback control system is necessary to correct for signal uncertainty (disturbances), model uncertainty, and instability [15], but it is out of scope of the present work. As a consequence, in all simulations exhibited here are without the feedback control system (i.e. open loop). For all cases, the long-stroke reference signal for the feedback controller can be obtained after defining $C_{\mathrm{ff}}(s)$ through (6), thus it is not presented further.

Reference signal. The fourth-order reference trajectory considered here ${ }^{2}$ is designed using the algorithm of [11] and depicted in Figure 4. The trajectory is $x_{\mathrm{r}} \in C^{3}(0, \infty)$, meaning it is smooth only up to its second derivative (acceleration), and its design is built on a sum of step signals in the snap signal $s_{\mathrm{r}}(t)$ by fulfilling limits in Table II. Specifically for the wafer stage application, the silicon wafer is exposed to the light only during time intervals of constant velocity, called scanning interval or procedure. Therefore, performance regarding accuracy is required mainly at these time frames.

\section{A. $C_{\mathrm{ff}-1}$ : Full long-stroke feedforward}

The stiff connection between the long-stroke table and the wafer table implies that any forces produced by the long-

\footnotetext{
${ }^{2}$ Specialized reference design can improve performance related to excitation of resonant modes [16], but it is out of scope of the presented work.
}

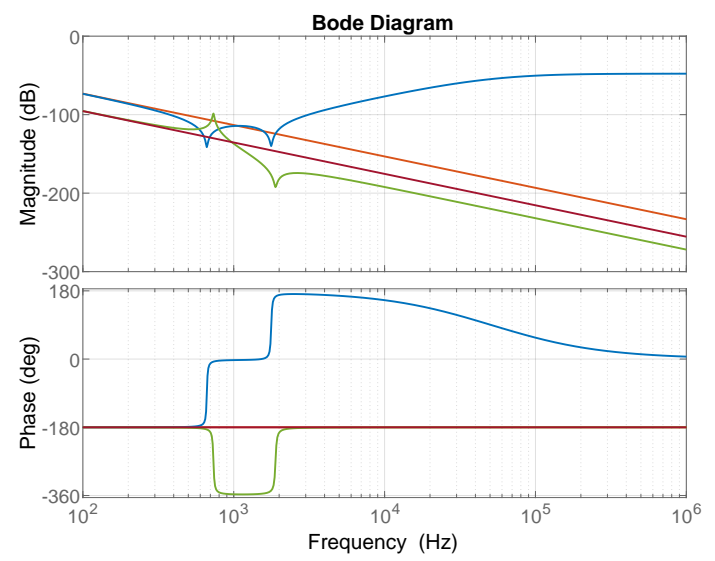

Fig. 5. Bode plot of each feedforward controller component multiplied by $1 / s^{4}$ (i.e. from the reference signal $s_{\mathrm{r}}(s)$ to the forces $F_{\mathrm{ls}}(s)$ and $F_{\mathrm{ss}}(s)$ ): $C_{\mathrm{ff}-\mathrm{ls}}(s)=\left(P_{\mathrm{wt}-\mathrm{ls}}(s)\right)^{-1}$ (blue curve), $C_{\mathrm{ff}-\mathrm{ls}}(s)=\left(m_{\mathrm{tot}} s^{2}\right)$ (orange curve), $C_{\mathrm{ff}-\mathrm{ss}}=C_{\mathrm{res}} C_{\mathrm{dc}-\text { gain }} s^{2}$ (green curve), $C_{\mathrm{ff}-\mathrm{ss}}=C_{\mathrm{dc}-\mathrm{gain}} s^{2}$ (purple red curve).

stroke actuator directly affects the position of the wafer table. Although moving the entire stage $(86 \mathrm{~kg})$ for performing the reference tracking does not seem an efficient idea in terms of energy consumption, the short-stroke actuator range is so limited $(15 \mu \mathrm{m})$ that the long-stroke table needs to approximately follow the trajectory anyway.

A trivial solution for balancing the contribution of each actuator for this system is then to have the long-stroke actuator performing all the positioning, leaving to the short-stroke actuator only the compensation of remaining errors by means of feedback control. For this concept, the feedforward force $F_{\mathrm{ss}}$ equals zero and hence $x_{\mathrm{wt}}=P_{\mathrm{wt}-1 \mathrm{~s}} C_{\mathrm{ff}-1 \mathrm{~s}} x_{\mathrm{r}}$. Therefore, $C_{\mathrm{ff}-\mathrm{ls}}=\left(P_{\mathrm{wt}-\mathrm{ls}}\right)^{-1}$ leads to perfect reference tracking and

$$
C_{\mathrm{ff}-1}(s)=\left[\begin{array}{ll}
0 & \left(P_{\mathrm{wt}-\mathrm{ls}}(s)\right)^{-1}
\end{array}\right]^{\top} .
$$

Observe from (3) that although the inverse is a non-causal filter with relative degree of 4 , the reference is a fourth order trajectory and we overcome this by substituting $x_{\mathrm{r}}=\frac{s_{\mathrm{r}}}{s^{4}}$ (Figure 5).

The resulting long-stroke force is depicted in Figure 6. Given that the snap reference signal is composed of a sum of step signals, which Laplace transform is $1 / s$, the initial value theorem analytically explains the instantaneous force changes of $6.32 \times 10^{4} \mathrm{~N}$ at each step of $s_{\mathrm{r}}(s)$. Physically, it means that the force impulses give the system an instantaneous acceleration change which compensates the compliant dynamics of the structure up to the moment the constant acceleration phase begins.

Although zero error is obtained, the demanded motion is physically impossible to be performed by the long-stroke actuator given its limitation to instantaneous changes in force. Furthermore, significant high-frequency amplification of the setpoint is present and may excite other highly undamped resonance modes that were not considered in the model.

An approach for dealing with these limitations is by designing $x_{\mathrm{r}}$ as a higher-order trajectory, but it leads to a considerable increase in the trajectory execution time and to a complex trajectory design. An alternative is to apply a simpler feedforward controller for the long-stroke mover, as presented in the next design. 


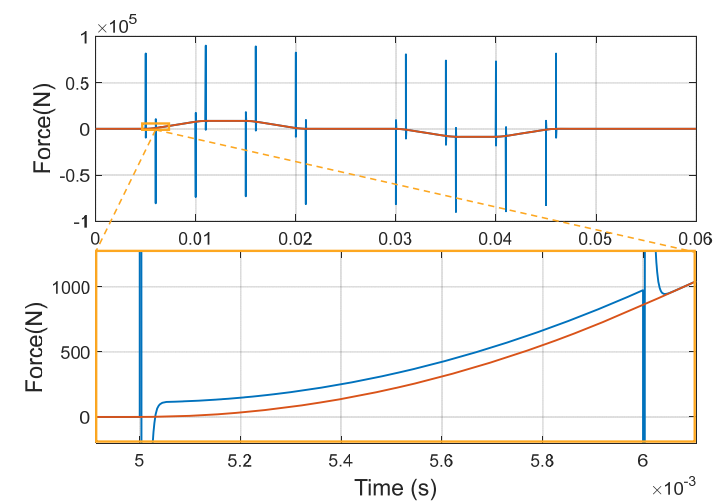

Fig. 6. $\quad F_{\mathrm{ls}}(t)$ generated by the feedforward controllers: $C_{\mathrm{ff}-\mathrm{ls}}=\left(P_{\mathrm{wt}-\mathrm{ls}}\right)^{-1}$ (blue curve), $C_{\mathrm{ff}-\mathrm{ls}}=\left(m_{\mathrm{tot}} s^{2}\right)$ (orange curve).

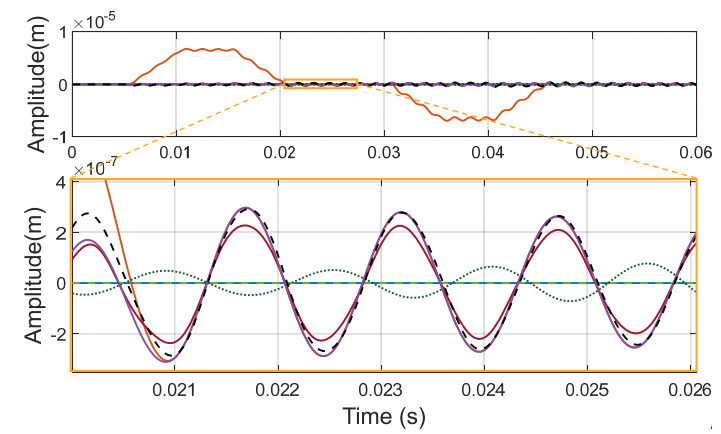

Fig. 7. Positioning error of the wafer table for each design: $C_{\mathrm{ff}-1}$ (blue solid curve), $C_{\mathrm{ff}-2}$ (orange solid curve), $C_{\mathrm{ff}-3}$ (dashed green curve), $C_{\mathrm{ff}-3-\text { mismatch }}$ (dark green dotted curve), $C_{\mathrm{ff}-4}$ (purple red solid curve), $C_{\mathrm{ff}-5}$ (purple solid curve), $C_{\mathrm{ff}-6}$ (black dashed curve).

\section{B. $C_{\mathrm{ff}-2}$ : Long-stroke rigid-body feedforward controller}

The considered concept of wafer stage provides high stiff connection between the tables and, consequently, a simplified yet accurate model for the stage is obtained by considering the full stage as a rigid body mass moved by the long-stroke actuator. For this simplified model, the plant inversion leads to $C_{\mathrm{ff}-\mathrm{ls}}=m_{\mathrm{tot}} s^{2}$, with $m_{\mathrm{tot}}=m_{\mathrm{wt}}+m_{\mathrm{ss}}+m_{\mathrm{ls}}$, and there is no short-stroke actuation $\left(C_{\mathrm{ff}-\mathrm{ss}}=0\right)$.

A feedforward controller for this simplified model is

$$
C_{\mathrm{ff}-2}(s)=\left[\begin{array}{ll}
0 & m_{\mathrm{tot}} s^{2}
\end{array}\right]^{\top},
$$

referred to as mass, acceleration or rigid body feedforward [11], [17]. It does not take into account the resonant nor the compliant dynamics of the plant ${ }^{3}$. Consequently, the piezoactuator and the connecting material are compressed during an increase of the acceleration, causing a positive error in the position of the wafer table (Figure 7). Furthermore, this input force excites the lightly damped resonance modes of the system, causing oscillation in the motion. Similar behavior is observed for a decrease in the acceleration.

Just including notch filters to $C_{\mathrm{ff}-2}$ makes the system deviate considerably from the reference $x_{\mathrm{r}}(t)$ and it was not presented here because the deviation surpasses the piezoactuator range capability.

\footnotetext{
${ }^{3}$ Refer to [17] for a more detailed explanation of the rigid body, compliant and resonant dynamics of the plant.
}

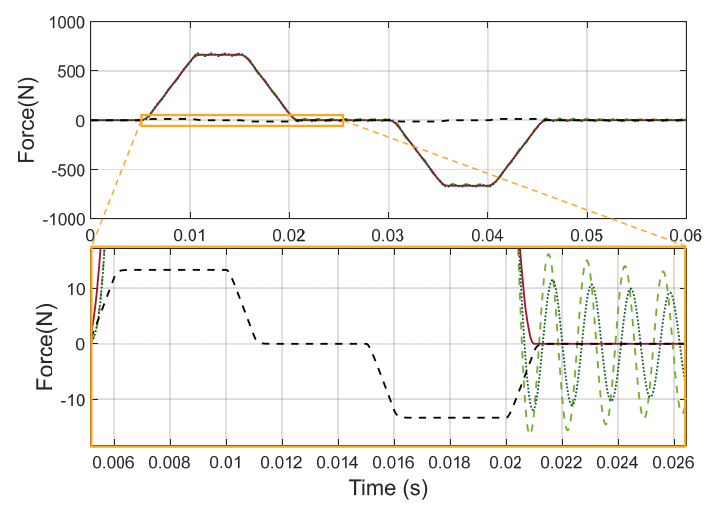

Fig. 8. $\quad F_{\mathrm{ss}}(t)$ generated by the following feedforward controllers: $C_{\mathrm{ff}-\mathrm{ss}}=$ $C_{\mathrm{res}} C_{\mathrm{dc}-\text { gain }} s^{2}$ (green dashed curve), $C_{\mathrm{ff}-\mathrm{ss}}=C_{\mathrm{res}-\text { mismatch }} C_{\mathrm{dc}-\mathrm{gain}} s^{2}$ (dark green dotted curve), $C_{\mathrm{ff}-\mathrm{ss}}=C_{\mathrm{dc}-\text { gain }} s^{2}$ (purple red solid curve), $C_{\mathrm{ff}-\mathrm{ss}}=$ $\left(1-C_{\text {trade-off }}\right) C_{\mathrm{dc}-\text { gain }} s^{2}$ (black dashed curve).

C. $C_{\mathrm{ff}-3}$ : Long-stroke rigid-body with complementary piezoactuator feedforward

For performance improvement, the piezo-actuator can be actively used in the feedforward control together with the long-stroke rigid body feedforward controller. By the sequential procedure, from (5) we obtain $C_{\mathrm{ff}-\mathrm{ss}}=C_{\mathrm{res}} C_{\mathrm{dc}-\text { gain }} s^{2}$ with

$$
C_{\mathrm{res}}=\left(\frac{\frac{m_{\mathrm{wt}} m_{\mathrm{ss}} m_{\mathrm{ll}} s^{2}+\left(c_{\mathrm{ss}-1 \mathrm{l}} m_{\mathrm{wt}}\left(m_{\mathrm{ss}}+m_{\mathrm{ls}}\right)+c_{\mathrm{p}} m_{\mathrm{ls}}\left(m_{\mathrm{wt}}+m_{\mathrm{ss}}\right)\right) s}{k_{\mathrm{ss}-1 \mathrm{~s}} m_{\mathrm{wt}}\left(m_{\mathrm{ss}}+m_{\mathrm{ls}}\right)+k_{\mathrm{p}} m_{\mathrm{ls}}\left(m_{\mathrm{wt}}+m_{\mathrm{ss}}\right)}+1}{\frac{m_{\mathrm{ss}} m_{\mathrm{ls}}}{\left(m_{\mathrm{ss}}+m_{\mathrm{ls}} k_{\mathrm{ss}}-\mathrm{ls}\right.} s^{2}+\frac{c_{\mathrm{ss}}-\mathrm{ls}}{k_{\mathrm{ss}}-\mathrm{ls}}+1}\right)
$$

and

$$
C_{\mathrm{dc}-\text { gain }}=m_{\mathrm{wt}}+\frac{k_{\mathrm{p}} m_{\mathrm{ls}}\left(m_{\mathrm{wt}}+m_{\mathrm{ss}}\right)}{k_{\mathrm{ss}-\mathrm{ls}}\left(m_{\mathrm{ls}}+m_{\mathrm{ss}}\right)} .
$$

Therefore, the feedforward controller

$$
C_{\mathrm{ff}-3}(s)=\left[\begin{array}{ll}
C_{\mathrm{res}}(s) C_{\mathrm{dc}-\mathrm{gain}} s^{2} & m_{\mathrm{tot}} s^{2}
\end{array}\right]^{\top}
$$

is another option that results in perfect tracking. By this design, the piezo-actuator applies forces not only to compensate its change in length caused by accelerations, but also to compensate the compression/elongation of the connecting material structure (compliance compensation, performed by $C_{\mathrm{dc}-\mathrm{gain}} s^{2}$ ). Additionally, it deals with the resonant dynamics by applying an oscillating force component which counteracts the resonant modes that are being excited by the long-stroke actuator force (resonant dynamics compensation, performed by $C_{\text {res }}$ ).

Although the piezo-actuator is capable of performing the needed forces, application of oscillating forces always needs to be analyzed carefully: a mismatch of the feedforward controller resonant peak leads to an oscillating force input that, instead of compensating, will be provoking oscillations. For instance, a discrepancy of only $20 \mathrm{~Hz}$ at the resonant peaks of $C_{\mathrm{ff}-3}(s)$ leads to a oscillatory force mismatch (Figure 8) that results in the error depicted in Figure 7.

\section{D. $C_{\mathrm{ff}-4}(s)$ : Short-stroke actuator compliance compensation}

A short-stroke feedforward controller that only compensates the compliant dynamics is given by $C_{\mathrm{ff}-\mathrm{ss}}=C_{\mathrm{dc}-\text { gain }} s^{2}$, resulting in

$$
C_{\mathrm{ff}-4}(s)=\left[\begin{array}{ll}
C_{\mathrm{dc}-\mathrm{gain}} s^{2} & m_{\mathrm{tot}} s^{2}
\end{array}\right]^{\top} .
$$


The piezo-actuator can smoothly compensate the compliance part, given that it is proportional to the acceleration and, thus, it is a smooth signal. The resulting error is then composed just by the excitation of resonant dynamics (see Figure 7).

\section{E. $C_{\mathrm{ff}-5}(s)$ : Standard compliance compensation}

For the wafer stage application, the silicon wafer is exposed to the light only during the scanning interval and only during these intervals the tracking error is required to be small. Therefore, the deviations caused by compliance during non-zero accelerations do not degrade printing performance. However, the transient response otherwise induced by the feedback controller compensating the compliant dynamic behavior can extend into the scanning interval and then affect performance.

A standard compliance compensation approach applied by the wafer scanning industry is to use $C_{\mathrm{c}}$ to manipulate the measured plant output (only in software) for masking the deformation of flexible dynamic structures during acceleration and deceleration. It prevents the feedback controller from acting on the compliance dynamics [4] and this manipulation can also be interpreted as a reference re-design at which the new reference $x_{\mathrm{r}-\text { red }}=\left(1+C_{\mathrm{c}}\right) x_{\mathrm{r}}$ contains the expected compliant dynamics and, thus, it does not generate errors for the feedback system.

The design procedure applied for this purpose is described in [17]. The long-stroke actuator feedforward component is given by the rigid body feedforward $\left(C_{\mathrm{ff}-\mathrm{ls}}=m_{\mathrm{tot}} s^{2}\right)$, there is no short-stroke actuator $\left(C_{\mathrm{ff}-\mathrm{ss}}=0\right)$ and the filter $C_{\mathrm{c}}$ is designed to have the compliant dynamic effect during accelerations as part of the reference signal. This leads to $C_{\mathrm{ff}-5}(s)=\left[\begin{array}{ll}0 & m_{\mathrm{tot}} s^{2}\end{array}\right]^{\top}$ and $C_{\mathrm{c}}=g_{\mathrm{cc}} s^{2}$ with

$$
g_{\mathrm{Cc}}=-\left(\frac{m_{\mathrm{wt}}\left(m_{\mathrm{ss}}+m_{\mathrm{ls}}\right)}{k_{\mathrm{p}} m_{\mathrm{tot}}}+\frac{m_{\mathrm{ls}}\left(m_{\mathrm{ss}}+m_{\mathrm{wt}}\right)}{k_{\mathrm{ss}-\mathrm{ls}} m_{\mathrm{tot}}}\right) .
$$

Notice that the system without feedback control operates exactly the same as with $C_{\mathrm{ff}-2}(s)$, just the considered positioning error is different. With this new error definition, which does not affect printing performance, approximately the same error as in $C_{\mathrm{ff}-4}$ is obtained, but without applying forces by the piezo-actuator.

For this adjusted reference, the acceleration reference is

$$
a_{\mathrm{r}-\mathrm{red}}=s^{2} x_{\mathrm{r}-\mathrm{red}}=s^{2}\left(1+g_{\mathrm{Cc}} s^{2}\right) x_{\mathrm{r}}=a_{\mathrm{r}}+g_{\mathrm{Cc}} s_{\mathrm{r}} .
$$

Thus, $a_{\mathrm{r}-\text { red }}(t)=a_{\mathrm{r}}(t)+g_{\mathrm{Cc}} s_{\mathrm{r}}(t)$ and it has step components for the fourth order reference signal (see Figure 9). The force reference for the wafer table is then proportional to this acceleration reference and the resultant abrupt force changes can lead to excitation of unmodeled resonant modes of the wafer table.

\section{F. $C_{\mathrm{ff}-6}(s)$ : (novel) mixed compliance compensation}

The passive change in piezo-actuator length for constant acceleration that is allowed by the design $C_{\mathrm{ff}-5}$ is equal to minus the piezo-actuator change in length in the design $C_{\mathrm{ff}-4}$, where the piezo-actuator is applied actively for compliance compensation. A combination of these two strategies makes

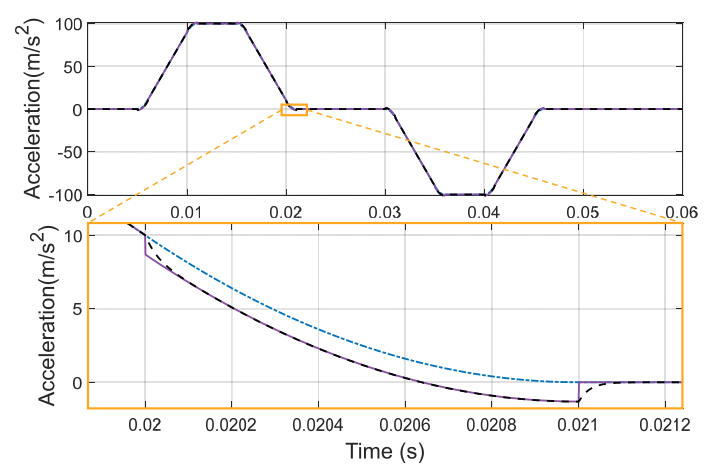

Fig. 9. Acceleration reference signals of the wafer table for feedback control: $a_{\mathrm{r}}$ (blue dot-dash curve), $a_{\mathrm{r}-\mathrm{red}}=s^{2}\left(1+g_{\mathrm{Cc}} s^{2}\right) x_{\mathrm{r}}$ (purple solid curve), $a_{\mathrm{r}-\mathrm{red}}=s^{2}\left(1+C_{\text {trade-off }} g_{\mathrm{Cc}} s^{2}\right) x_{\mathrm{r}}$ (black dashed curve)

it possible to maintain the fourth-order reference trajectory, with smoother final reference trajectory and by employing more efficiently the feedforward piezo-actuator force. This combination lead to a novel feedforward controller and constitutes Contribution C3 and is presented next.

The proposed controller is given by

$$
C_{\mathrm{ff}-6}(s)=\left[\begin{array}{ll}
\left(1-C_{\text {trade-off }}\right) C_{\mathrm{dc}-\text { gain }} s^{2} & m_{\mathrm{tot}} s^{2}
\end{array}\right]^{\top},
$$

with reference filter adjustment $C_{\mathrm{c}}=C_{\text {trade-off }} g_{\mathrm{cc}} s^{2}$. The $C_{\text {trade-off }}$ is a tunable component that defines how much of the compliance dynamics is compensated by the reference redesign and, in a complementary way, how much it is compensated by the short-stroke actuator forces ${ }^{4}$. We adopt

$$
C_{\text {trade }- \text { off }}=\frac{1}{s / \alpha+1},
$$

where $\alpha \in \mathbb{R}$ is a tunable parameter that determine transition smoothness and duration from piezo-actuator to reference compliance compensation. For the considered example, we use $\alpha=2 \cdot 10^{4}$ and obtain the acceleration profile $a_{\mathrm{r}-\text { red }}$ smooth transition from decelerating to zero acceleration, as depicted in Figure 9, with transition duration of less than $0.5 m \mathrm{~s}$. The filter $C_{\text {trade-off }}$ generates $C_{\mathrm{ff}-\mathrm{ss}}=(s+$ $\alpha)^{-1} C_{\mathrm{dc}-\text { gain }} s^{3}$, meaning that zero force is applied by the piezo-actuator during constant acceleration (after the filter transient is passed), see Figure 8.

\section{G. Comparison and remarks}

Six feedforward control systems are presented in this section and Table III contains a summary of the main characteristics of these systems.

If performance for tracking $x_{\mathrm{r}}(t)$ were to be achieved at all time, the design $C_{\mathrm{ff}-4}$ option combined to a feedback control system and possibly reference redesign to avoid excitation of resonant modes or design $C_{\mathrm{ff}-1}$ with a higher reference trajectory order and feedback system are the best options. The choice on which one is better for a specific application lies mainly on the analysis of the trade-off between the increase in reference trajectory order and the application of piezo-forces during non-constant accelerations.

\footnotetext{
${ }^{4}$ Observe that for $C_{\text {trade-off }}=0$ the overall feedforward control system is equal to $C_{\mathrm{ff}-4}$ and for $C_{\text {trade-off }}=1$ to $C_{\mathrm{ff}-5}$.
} 
TABLE III

COMPARISON OF FEEDFORWARD CONTROLLERS FOR THE REFERENCE TRAJECTORY DEPICTED IN FIGURE 4

\begin{tabular}{|c|c|c|c|c|c|c|}
\hline & $C_{\mathrm{ff}-1}(s)$ & $C_{\mathrm{ff}-2}(s)$ & $C_{\mathrm{ff}-3}(s)$ & $C_{\mathrm{ff}-4}(s)$ & $C_{\mathrm{ff}-5}(s)$ & $C_{\mathrm{ff}-6}(s)$ \\
\hline Perfect reference tracking & $\checkmark$ & $x$ & $\checkmark$ & $x$ & $\times$ & $\times$ \\
\hline Simulation error less than $1 \mu \mathrm{m}$ & $\checkmark$ & $x$ & $\checkmark$ & $\checkmark$ & $\checkmark$ & $\checkmark$ \\
\hline Conform limits of the actuators & $\times$ & $\checkmark$ & $\checkmark$ & $\checkmark$ & $\checkmark$ & $\checkmark$ \\
\hline Piezo-actuator feedforward forces while scanning & $x$ & $x$ & $\checkmark$ & $\checkmark$ & $\times$ & $\times$ \\
\hline Feedforward oscillating forces & $x$ & $\times$ & $\checkmark$ & $x$ & $\times$ & $\times$ \\
\hline Applicable to any $4 t h$ order reference trajectory & $\checkmark$ & $\checkmark$ & $\checkmark$ & $\checkmark$ & $x$ & $\times$ \\
\hline
\end{tabular}

Design $C_{\mathrm{ff}-6}$ is the more attractive for the application in focus. Piezo-actuators feedforward forces are only employed in non-constant acceleration instants and with relatively low forces, being the maximum value of this force easily tuned by the parameter $\alpha$. The new acceleration reference signal is continuous on time and can also be tuned through $\alpha$. The final positioning error is just regarding resonant dynamics that, as mentioned, should be compensated by other means.

\section{CONCLUSION}

The main contributions of this paper are the sequential feedforward controller design procedure, the investigation of different feedforward control designs and the proposed mixed compliance compensation controller. The sequential procedure can be applied directly for the controller's design, but can also be used as a start-point for more advanced synthesis methods. In addition, the novel proposed feedforward controller synthesis is efficient for scanning applications: the employment of feedforward piezo-actuator forces only during transitions between constant and varying acceleration intervals reserves the piezo-actuator forces to the feedback control system while not contributing to excitation of resonant modes of the wafer table.

Next step includes the application of the proposed feedforward controller to a real setup. For this, a discrete time controller version needs to be obtained, taking into account discretization and quantization effects. In addition, a proper amplifier needs to be designed to deal with hysteresis present in the piezoelectric effect and also the expected piezomaterial compression effects in the electrical domain. Future research goals include analysis of the effects of different uncertainties types and enhancement of the proposed design technique by transforming it into an optimization problem. Expansion to stages with more degrees of freedom is also envisioned.

\section{ACKNOWLEDGMENT}

This work is part of the TU/e-ASML mini Impulse program "Advanced piezo-electric wafer stage for lithography and metrology".

\section{REFERENCES}

[1] G. E. Moore et al., Cramming more components onto integrated circuits. McGraw-Hill New York, 1965.

[2] Intel company. Over 50 years of moore's law. [Online]. Available: https://www.intel.com/content/www/us/en/siliconinnovations/moores-law-technology.html
[3] H. Butler, "Position control in lithographic equipment: applications of control," IEEE Control Systems Magazine, vol. 31, no. 5, pp. 28-47, 2011.

[4] M. F. Heertjes, H. Butler, N. Dirkx, S. van der Meulen, R. Ahlawat, K. O'Brien, J. Simonelli, K. Teng, and Y. Zhao, "Control of wafer scanners: Methods and developments," in 2020 American Control Conference (ACC). IEEE, 2020, pp. 3686-3703.

[5] A. Mitrovic, K. K. Leang, and G. M. Clayton, "Analysis and experimental comparison of range-based control for dual-stage nanopositioners," Mechatronics, vol. 69, p. 102371, 2020.

[6] J. Holterman and W. Groen, An Introduction to piezoelectric materials and applications. Stichting Applied Piezo, 2013.

[7] M. Heertjes, D. Hennekens, and M. Steinbuch, "MIMO feed-forward design in wafer scanners using a gradient approximation-based algorithm," Control Engineering Practice, vol. 18, no. 5, pp. 495-506, 2010.

[8] L. Blanken, F. Boeren, D. Bruijnen, and T. Oomen, "Rational iterative feedforward tuning: Approaches, stable inversion, and experimental comparison," in 2016 American Control Conference (ACC). IEEE, 2016, pp. 2629-2634.

[9] S. H. van der Meulen, R. L. Tousain, and O. H. Bosgra, "Fixed structure feedforward controller design exploiting iterative trials: Application to a wafer stage and a desktop printer," 2008.

[10] J. Lunenburg, "Inversion-based mimo feedforward design beyond rigid body systems," Eindhoven University of Technology, Tech. Rep., 2010.

[11] P. Lambrechts, M. Boerlage, and M. Steinbuch, "Trajectory planning and feedforward design for electromechanical motion systems," Control Engineering Practice, vol. 13, no. 2, pp. 145-157, 2005.

[12] "IEEE standard on piezoelectricity," ANSI/IEEE Std 176-1987, 1988.

[13] H. Adriaens, W. L. De Koning, and R. Banning, "Modeling piezoelectric actuators," IEEE/ASME Transactions on mechatronics, vol. 5, no. 4, pp. 331-341, 2000.

[14] T. Castenmiller, F. van de Mast, T. de Kort, C. van de Vin, M. de Wit, R. Stegen, and S. van Cleef, "Towards ultimate optical lithography with NXT: 1950i dual stage immersion platform," in Optical Microlithography XXIII, vol. 7640, 2010, p. 76401.

[15] S. Skogestad and I. Postlethwaite, Multivariable feedback control: analysis and design. Wiley New York, 2007, vol. 2.

[16] L. Biagiotti, C. Melchiorri, and L. Moriello, "Optimal trajectories for vibration reduction based on exponential filters," IEEE Transactions on Control Systems Technology, vol. 24, no. 2, pp. 609-622, 2015.

[17] N. Kontaras, M. Heertjes, and H. Zwart, "Continuous compliance compensation of position-dependent flexible structures," IFACPapersOnLine, vol. 49, no. 13, pp. 76-81, 2016. 